NASA-CR-204546

\title{
The Cause of the Hot Spot in Vegetation Canopies and Soils: Shadow-Hiding Versus Coherent Backscatter
}

\author{
Bruce Hapke, ${ }^{*}$ Dominick DiMucci, ${ }^{*}$ Robert Nelson, ${ }^{\dagger}$ \\ and William Smythe ${ }^{\dagger}$
}

\begin{abstract}
Two different mechanisms, shadow-hiding and coherent backscatter, can cause a hot spot, or opposition effect, in the bidirectional reflectance of vegetation and soils. Because the two mechanisms sample different properties, it is important to know which one is primarily responsible in a given medium. This question can be answered by measuring the bidirectional reflectance in circularly polarized light. If the results of the limited experiments reported here can be extrapolated to a wider range of materials, it appears that the primary cause of the hot spot in most vegetation canopies and in moist, clumpy soils is shadow-hiding. However, in vegetation with large numbers of wavelength-sized structures, such as mosses, and in dry, fine-grained soils, the hot spot is dominated by coherent backscatter.
\end{abstract}

\section{INTRODUCTION}

The surge in the brightness of a surface viewed in the backscatter direction has been known for over a century. It was first discovered by Seeliger $(1887 ; 1895)$ in light scattered from the rings of Saturn. It was independently rediscovered in light from asteroids and the moon by Gehrels (Gehrels, 1956; Gehrels et al., 1964), who called it the "opposition effect" because it occurs near astronomical opposition when the phase angle (the angle between the sun and the viewer as seen from the surface of the object) approaches zero. It is also sometimes

*Department of Geology and Planetary Science, University of Pittsburgh

${ }^{\dagger}$ Jet Propulsion Laboratory, Pasadena

Address correspondence to Bruce Hapke, 321 Old Engineering Hall, Univ. of Pittsburgh, Pittsburgh, PA 15260.

Received 30 June 1995; revised 29 November 1995 called the "heiligenschein," and was first reported in light scattered from particulate surfaces in terrestrial laboratories by Oetking (1966). In vegetation canopies the opposition effect is known as the "hot spot."

Ever since Seeliger's discovery, the accepted explanation for the opposition effect, or hot spot, has been shadow-hiding, in which elements of the medium hide their own shadows at zero phase angle. However, it has recently been recognized that an interference phenomenon known as coherent backscatter can cause an opposition effect. Kuga and Ishimaru (1984) were the first to invoke this mechanism to explain the backscatter peak they observed in the bidirectional reflectance of a particulate medium. It was shown that this process is responsible for the opposition effect of the moon (Hapke et al., 1993) and, very likely, of most of the other bodies in the solar system. Because the hot spot is a useful tool in the remote sensing of vegetation canopies and soils, and because the two types of mechanisms sample different properties of a medium, it is important to understand which is the primary cause of the hot spot. In this paper we report the results of measurements that enable the two phenomena to be distinguished in selected samples of vegetation and soil.

\section{MECHANISMS OF THE HOT SPOT}

The production of a surge in the brightness of a medium in the backscatter direction by shadow-hiding is intuitively obvious and is illustrated in Figure 1. If the medium consist of scatterers that are large compared with the wavelength of the incident light, they will cast well-defined shadows on other parts of the medium. These shadows are partly visible when the surface is viewed from any direction except the direction of the 


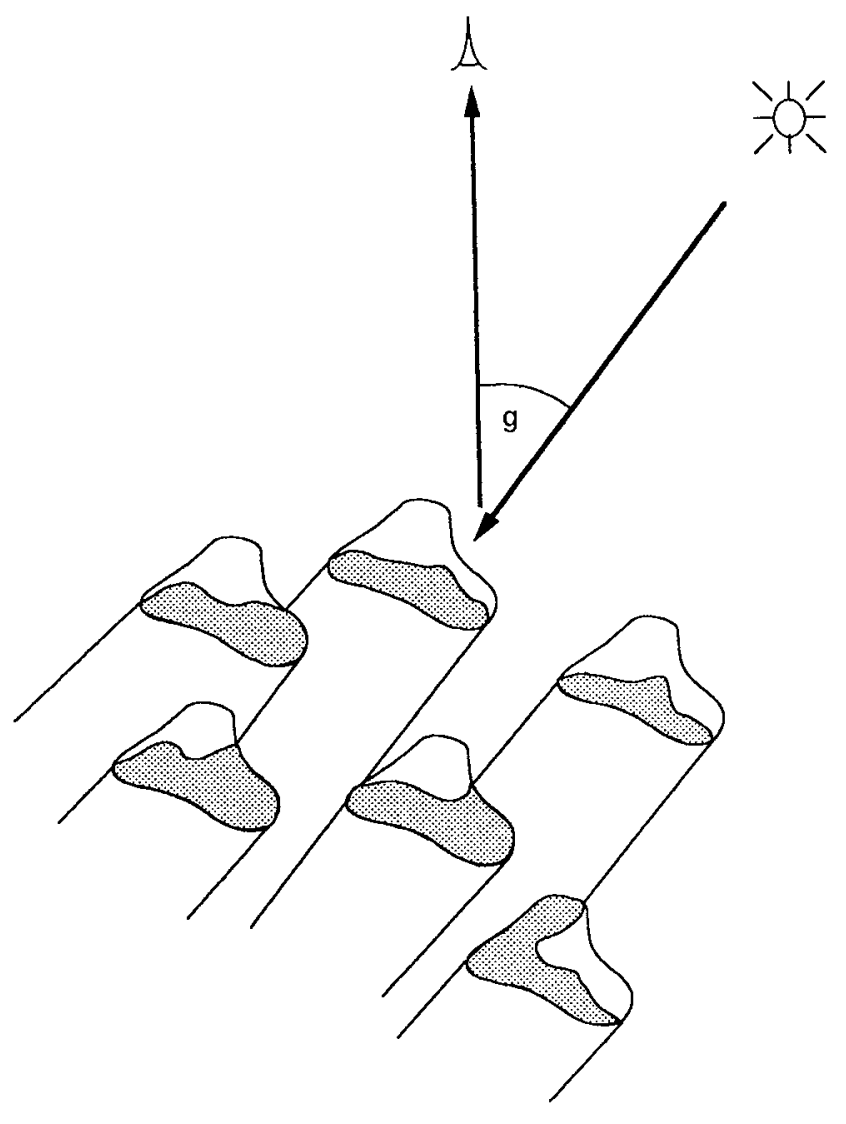

Figure 1. Schematic diagram of the shadow-hiding mechanism of the hot spot.

source of incident light; at this special angle each scatterer hides its own shadow. The hot spot involves the rays of light that are scattered only once from the leaves of a canopy or grains of a soil. The multiply scattered light serves mainly to fill in the shadows and decrease the amplitude of the peak relative to the continuum reflectance. The angular width of the peak depends on the porosity and size distribution of the scatterers of the medium (Hapke, 1986; Hapke et al., 1993).

By contrast, coherent backscatter is a constructive interference phenomenon that occurs when the medium contains abundant scatterers that are of the order of the wavelength in size and are separated by similar distances. It depends intrinsically on the wave nature of light.

The mechanism is illustrated in Figure 2. The line $U-S_{N}$ represents a wavefront of light incident on a medium made up of objects that scatter light, denoted by $S_{0}, S_{1}, \ldots$ A ray $A-S_{0}$ associated with wavefront $U-S_{N}$ (perpendicular to $A-S_{0}$ ) strikes $S_{0}$ and is scattered to $S_{1}$, then to $S_{2} \ldots, S_{N-1}$, and finally to $S_{N}$, from which it exits the medium along path $S_{N}-A^{\prime}$ in a direction that makes an angle $g$ with the incoming light. The exiting portion

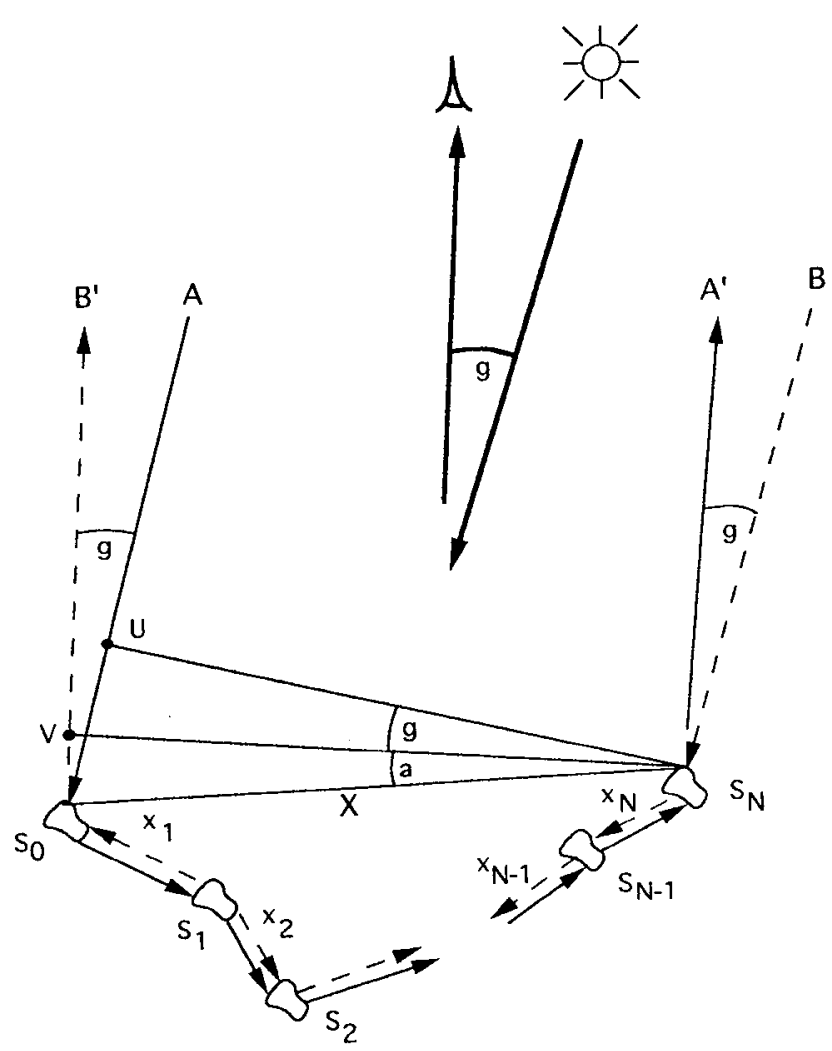

Figure 2. Schematic diagram of the coherent backscatter mechanism of the hot spot.

of the ray, $S_{N}-A^{\prime}$, contributes to the wavefront $V-S_{N}$ (perpendicular to $S_{N}-A^{\prime}$ ) scattered into phase angle $g$ by the medium. Now, for every ray $A-A^{\prime}$, a ray $B-B^{\prime}$ exists that is also associated with the incident wavefront $U-S_{N}$ and that traces the same path as $A-A^{\prime}$ inside the medium, but in reverse. That is, it is incident along path $B-S_{N}$ (parallel to $A-S_{0}$ ), is scattered to $S_{N-1}, \ldots$, to $S_{1}$, and finally to $S_{0}$, from which it exits along path $S_{0}-B^{\prime}$ (parallel to $S_{N^{\prime}}-A^{\prime}$ ), and also contributes to the scattered wavefront $V-S_{N}$.

Let the distance between $S_{j-1}$ and $S_{j}$ be $x_{j}$, and let $X$ be the direct distance between $S_{0}$ and $S_{N}$. After emerging from the medium, ray $A-A^{\prime}$ has traveled a total distance from $U$ to $S_{N}$,

$$
X_{\mathrm{A}}=X \sin (a+g)+x_{1}+x_{2}+\ldots+x_{N},
$$

while during the same time interval ray $B-B^{\prime}$ has traveled a distance

$$
X_{B}=X_{N}+\ldots+x_{2}+x_{1}+X \sin a .
$$

The path difference is

$$
\Delta X=X_{A}-X_{B}=X[\sin (a+g)-\sin a] \approx X g \cos a,
$$

if $g<<1$. The phase difference associated with this path difference is

$$
\Delta \varphi=\frac{2 \pi}{\lambda} \Delta X \approx \frac{2 \pi}{\lambda} X g \cos a .
$$


At the exact backscatter direction, $g=0$, so $\Delta \varphi=0$ and the two parts of the wavefront interfere constructively. Suppose the amplitudes of the electric fields associated with each ray after the final scattering is $E$. If $\Delta \varphi$ is random, their combined intensities are proportional to $|E|^{2}+|E|^{2}=2|E|^{2}$. However, at zero phase angle the amplitudes of the waves add coherently, so that the combined intensities are proportional to $|E+E|^{2}=4|E|^{2}$; thus, the scattered intensity is doubled at this special angle. The coherence is effectively lost when $\Delta \varphi \geqslant 1$. Thus, the coherence causes a peak in the scattered intensity whose angular width (in radians) is seen from Eq. (1) to be approximately $\Delta g \approx \lambda / 2 \pi X \cos$ a.

Although this simplified derivation is only approximate, it demonstrates the general nature of the production of an opposition effect by coherent backscatter. Note that the phenomenon involves only light that has been scattered two or more times within the medium. More general and rigorous theoretical treatments (Stephen and Cwilich, 1986; MacKintosh and John, 1988; Peters, 1992) shows that this simple model overestimates the amplitude of the hot spot, and also that

$$
\Delta g=b \lambda / 2 \pi D,
$$

where $D$ is the mean, random-walk, path length of photons in the medium and $b$ is a constant, whose empirical value is $b=0.72$ (Van Albada et al., 1987).

Another shortcoming of the simplified derivation given here is that it does not take account of polarization. If the incident radiation is linearly polarized, it turns out that the direction of polarization tends to be preserved in both the shadow-hiding and coherent backscatter processes. Hence, the opposition effect is much stronger in light for which the direction of polarization is the same as that of the incident illumination than in light of the orthogonal direction of polarization (Wolf and Maret, 1985; Van Albada and Lagendik, 1985; Van Albada et al., 1987).

However, if the incident light is circularly polarized and the medium is observed with detectors sensitive to the handedness or helicity of the light, the response will be different, depending on the nature of the process causing the opposition effect. If the opposition effect is caused by shadow-hiding, its amplitude will be larger when observed in the opposite helicity as the incident light, while if it is caused by coherent backscattering, it will be stronger when observed in the same helicity (MacIntosh and John, 1988; Hapke, 1990; Hapke and Blewett, 1991).

This provides a way of determining which type of process dominates the brightness surge in a given material. The medium is illuminated with circularly polarized light and the reflectance as a function of angle is measured in the same and opposite helicities as the incident light. The circular polarization ratio $\mu_{C}$ is then calculated as a function of phase angle $\mathrm{g}$, where $\mu_{C}(\mathrm{~g})$ is defined as the ratio of the intensity of the light scattered with the same helicity as the incident light to the intensity of the light scattered with the opposite helicity. If the opposition surge is caused by shadowhiding, $\mu_{C}$ will decrease as $g \rightarrow 0$, while if it is caused by coherent backscatter, $\mu_{C}$ will increase.

\section{EXPERIMENTAL}

\section{Apparatus}

The measurements were done using the goniometric photopolarimeter in the Planetary Surfaces Laboratory at the University of Pittsburgh. This instrument was described in Hapke and Wells (1981) and Woessner and Hapke (1987). It uses a movable quartz-halogen incandescent lamp source and a movable photomultiplier with an S-20 response as detector, plus appropriate collimating and focusing lenses. All measurements were made with the surface observed vertically, while the direction of illumination varied between $1^{\circ}$ and $30^{\circ}$ from the zenith. After leaving the source, tile light passes through a polarizing filter with its axis oriented parallel to the plane containing the directions to the source and detector. A quarter-wave plate placed just above the target converts the polarization of the incident light from linear to circular and the polarization of the light scattered by the target from circular back to linear. A rotatable polarizing filter in front of the detector distinguishes the two directions of polarization. The instrument can make measurements at phase angles as small as $1^{\circ}$, with an angular resolution of about $0.5^{\circ}$.

The measurements were made in white light with no wavelength filter in the optical train. Although the instrument is sensitive to a range of wavelengths from approximately $350 \mathrm{~nm}$ to $820 \mathrm{~nm}$, more than $97 \%$ of the signal comes from the visible, $400-700 \mathrm{~nm}$, and peaks in the red. Hence, even though the quarter wave plate is not effective outside this range, this has only a minor effect on the measured values of $\mu_{C}$. Also, it is the change in $\mu_{C}$ as $g \rightarrow 0$ that is of interest, rather than the absolute value of $\mu_{C}$, and the lack of UV and IR response will not affect this.

\section{Samples}

The choice of vegetation was dictated by the requirements that the vegetation be common in western Pennsylvania and that the leaves be smaller than the field of view of the instrument, about $2.5 \mathrm{~cm}$. Hence, we chose white or Dutch clover, lawn grass, spruce needles and ground moss. The clover was Trifolium repens. The species of grass was uncertain; however, in the northeastern United States most lawns are mixtures of bluegrass, fescue, and rye. The blades were about $5 \mathrm{~cm}$ long. The moss was of order Bryales, but the exact species was uncertain. The clover, grass, and moss were alive 


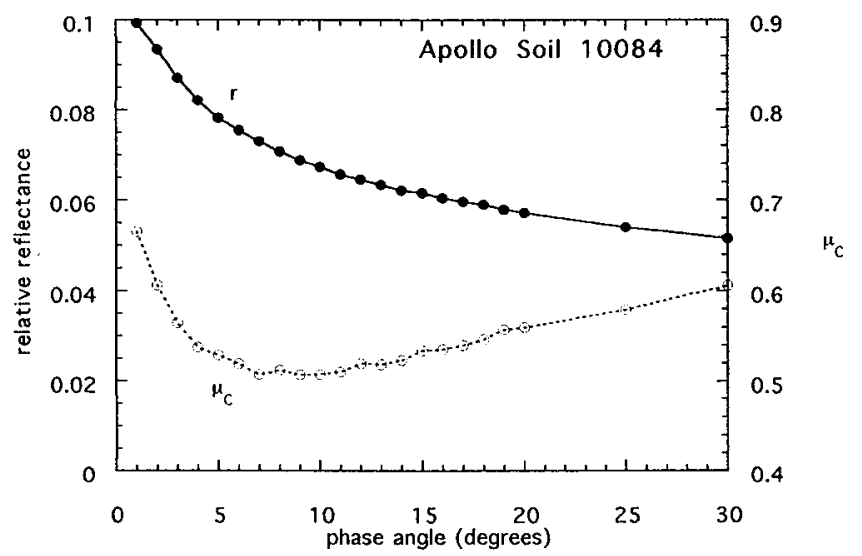

Figure 3. Relative reflectance and circular polarization ratio of Apollo lunar soil sample 10084.

and still growing in soil. The spruce needles were freshly removed from their tree and arranged to form a thick mat of horizontal randomly oriented needles.

We also studied the soil of the lawn in which the clover grew in two conditions: moist as freshly dug, and after drying undisturbed for 5 days at room temperature. For comparison with a material known to exhibit coherent backscatter, we also measured a sample of Apollo lunar soil, NASA No. 10084.

\section{RESULTS}

The relative reflectance $r$ and circular polarization ratio $\mu_{C}$ are plotted as a function of phase angle $g$ in Figures $3-8$. The errors in the relative intensities are less than $\pm 1 \%$, and in the circular polarization ratios, about \pm 0.01 . The scatter in the data is caused mainly by the fact that the nonuniformities in the sample are not small compared with the size of the area seen by the detector.

Figure 3 shows the data for the lunar soil. These curves are typical of a dry, fine-grained soil having

Figure 4. Relative reflectance and circular polarization ratio of a clump of live clover.

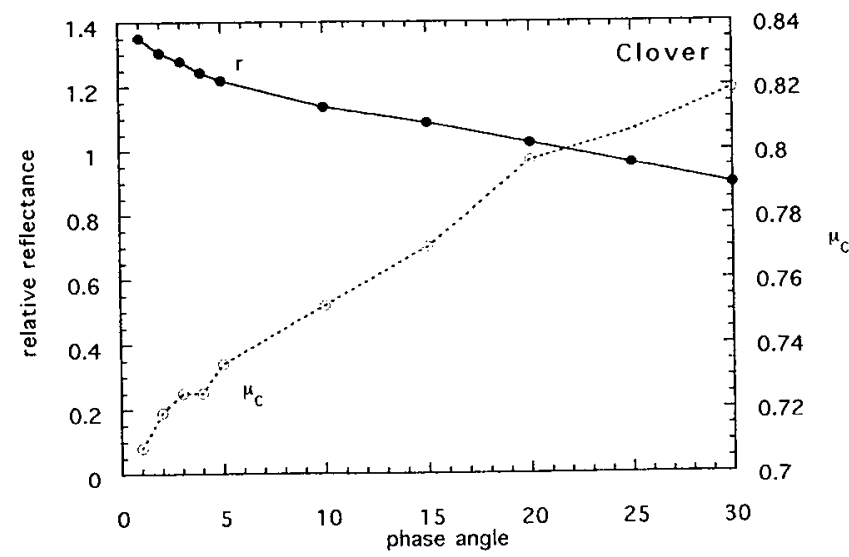

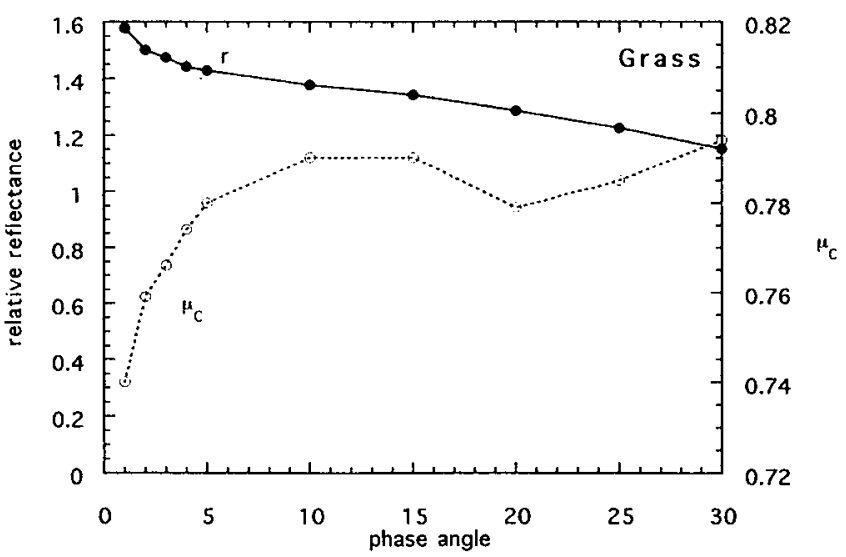

Figure 5. Relative reflectance and circular polarization ratio of a clump of live grass.

an exceptionally strong coherent backscatter opposition effect (Hapke et al., 1993). Note the strong surge in brightness about $7^{\circ}$ wide near zero phase, in which $\mu_{C}$ increases with decreasing $g$.

Similar curves are given for the clump of clover in Figure 4, for the grass in Figure 5 and for the spruce needles in Figure 6. Although each of these samples exhibits a hot spot, $\mu_{C}$ decreases monotonically as $g$ decreases. Thus, it may be inferred that for these vegetations the hot spot is due primarily to shadow-hiding. However, in Figure 7 for moss, it is seen that $\mu_{C}$ increases with decreasing $g$ when $g<4^{\circ}$. Hence, the hot spot of the moss appears to be caused primarily by coherent backscatter.

Curves for the soil in which the clover grew are given in Figure 8. As collected, this sample was damp, but not wet, and had many millimeter-sized clumps. Figure 8 shows that in this condition the opposition effect of the soil is caused by shadow-hiding. However, after the soil was allowed to dry it exhibited coherent backscatter.

Figure 6. Relative reflectance and circular polarization ratio of a carpet of spruce needles.

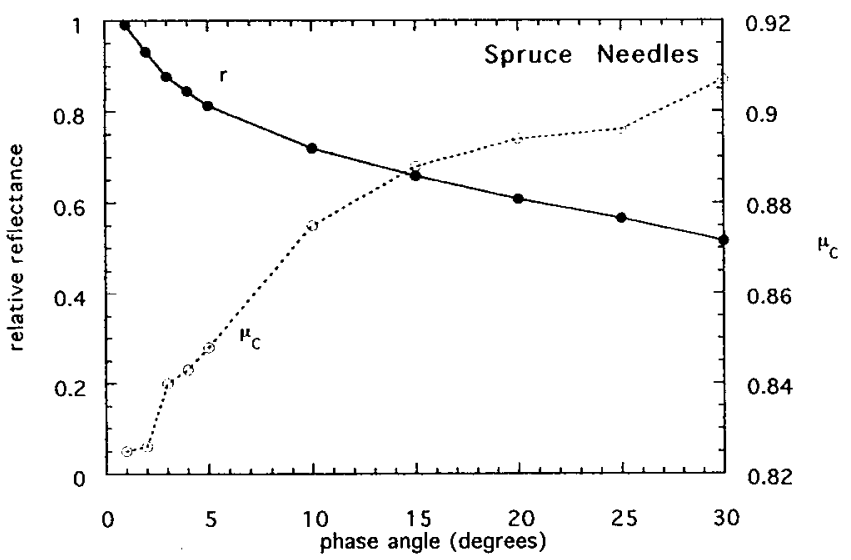




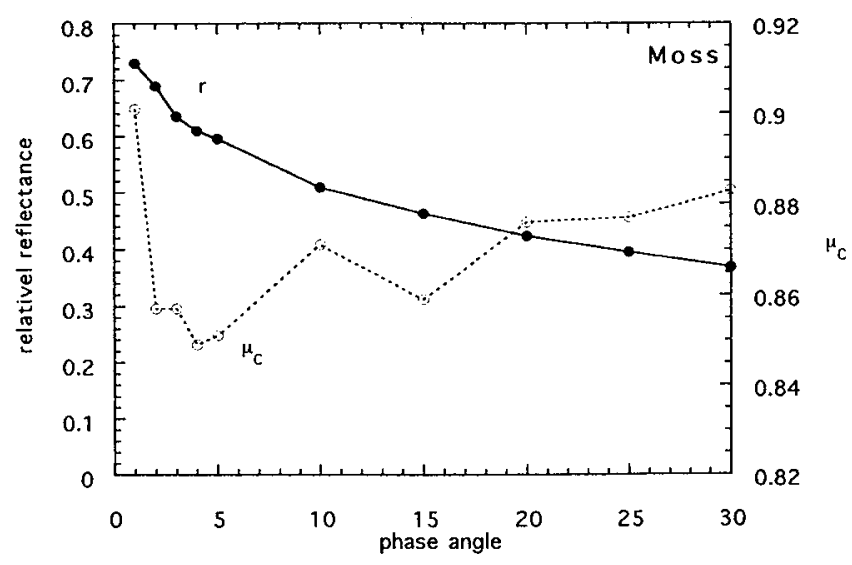

Figure 7. Relative reflectance and circular polarization ratio of live ground moss.

\section{DISCUSSION}

An opposition effect or hot spot caused by shadowhiding requires a material that consists of shadowcasting objects much larger than the wavelength at which the observations are made. A shadow-hiding hot spot involves only light that has been scattered once from a leaf or grain of soil, and the angular width depends on the porosity of the medium. By contrast, a wide coherent backscatter hot spot requires abundant scatterers not too much larger than the wavelength, separated by similar distances. If the mean separation is much larger than the wavelength, the brightness surge will be too narrow to be observed. This type of hot spot involves only multiply scattered light, and the angular width depends on the ratio of the wavelength to the average distance a photon travels between scatterings in the medium.

The two mechanisms can be distinguished by mea-

Figure 8. Relative reflectances and circular polarization ratios of the soil in which the clover of Figure 4 grew in the damp condition as collected (circles) and after drying in air for 5 days (triangles).

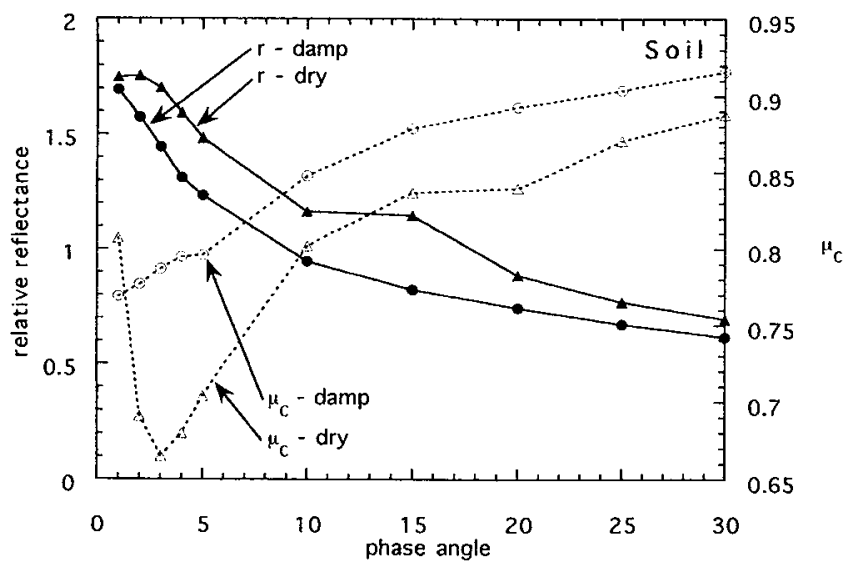

suring the bidirectional reflectance in circularly polarized light. Because of the necessity of using circularly polarized illumination, it would be difficult to extend these studies to field measurements on large scale vegetation, such as forest canopies, although this could be done at night using artificial illumination. Hence, even though these results are from a limited pilot study, it is hoped that they are sufficiently general to indicate the natures of the hot spots observed in remote measurements of the Earth's surface.

If our results can be generalized, they imply that, in visible light, the wide hot spot observed in most vegetation (including broad-leafed and coniferous) canopies and in moist, clumpy soils is probably caused mainly by shadow-hiding. It was not obvious $a$ priori that this would be the case, because vegetation contains wavelength-sized structures, such as cells, and most soils contain small clay-sized particles, both of which might have caused coherent backscatter.

Evidently, there is insufficient multiple scattering between the microscopic structures within the leaves and needles to cause appreciable coherent backscatter. Similarly, the presence of water in the pores of the moist soil apparently reduces the contrast in refractive index between the grains of soil and their environment sufficiently to inhibit appreciable multiple scattering. When the soil is dried and the pore water is removed, coherent backscatter becomes important. Lunar soil, which is inherently fine-grained and dry, exhibits an exceptionally strong coherent backscatter hot spot. The coherent backscatter nature of the hot spot of moss is probably due to the presence of abundant spores and other small structures on the visible surfaces.

The preliminary results of this paper imply that current hot spot models that invoke shadow-hiding (e.g., Verstraete et al., 1990; Borel et al., 1991; Kuusk, 1991; Hapke, 1986) are probably correct for most vegetation canopies and moist, clumpy soils in the visible region of the spectrum. However, coherent backscatter may dominate the hot spot in certain scenes where there are abundant wavelength-sized scatterers, such as mosses and dry, fine-grained soils.

Although both shadow-hiding and coherent backscatter can occur in the same medium, usually one mechanism dominates in the hot spot. Vegetation has a low albedo in the visible, which allows shadow-hiding by the leaves to be the most important. However, plants have high albedos in the near infrared, which may allow coherent backscatter between internal elements of the leaves to dominate there. Further studies will be required to determine which process is the primary cause of the hot spot at longer wavelengths.

This research was supported by grants from the Planetary Geology and Geophysics Program of the National Aeronautics and Space Administration. 


\section{REFERENCES}

Borel, C., Gerstl, S., and Powers, B. (1991), The radiosity method in optical remote sensing of structured 3-D surfaces, Remote Sens. Environ. 36:13-44.

Gehrels, T. (1956), Photometric studies of asteroids. V. The lightcurve and phase function of 20 Massalia, Astrophys. $J$. 123:331-338.

Gehrels, T., Coffeen, D., and Owings, D. (1964), Wavelength dependence of polarization. III. The lunar surface, Astron. J. 69:826-852.

Hapke, B. (1986), Bidirectional reflectance spectroscopy, 4. The extinction coefficient and the opposition effect, Icarus 67:264-280.

Hapke, B. (1990), Coherent backscatter and the radar characteristics of outer planet satellites, Icarus 88:407-417.

Hapke, B., and Wells, E. (1981), Bidirectional reflectance spectroscopy. 2. Experiments and observations, J. Geophys. Res. 86:3055-3060.

Hapke, B., and Blewett, D. (1991), Coherent backscatter model for the unusual radar reflectivity of icy satellites, Nature 352:46-47.

Hapke, B., Nelson, R., and Smyth, W. (1993), The opposition effect of the moon: the contribution of coherent backscatter, Science 260:509-511.

Kuga, Y., and Ishimaru, A. (1984), Retroreflection from a dense distribution of spherical paticles, J. Opt. Soc. Am. A1:831-835.

Kuusk, A. (1991), The hot spot effect in plant canopy reflectance, in Photon-Vegetation Interactions (R. Myneni and J. Ross, Eds.), Springer-Verlag, New York, pp. 139-159.

MacKintosh, F., and John, S. (1988), Coherent backscattering of light in the presence of time-reversal, noninvariant and parity-violating media, Phys. Rev. B 37:1884-1897.

Oetking, P. (1966), Photometric studies of diffusely reflecting surfaces with applications to the brightness of the moon, J. Geophys. Res. 71:2505-2413.

Peters, K. (1992), Coherent-backscatter effect: a vector formulation accounting for polarization and absorption effects and small or large scatterers, Phys. Rev. B. 46:801-812.

Seeliger, H. (1987), Zur Theorie der Beleuchtung der grossen Planeten inbesondere des Saturn, Abhandl. Bayer. Akad. Wiss. Math-Naturw. Kl. II 16:405-516.

Seeliger, H. (1895), Theorie der Beleuchtung staubformiger kosmischen Masses insbesondere des Saturinges, Abhandl. Bayer. Akad. Wiss. Math-Naturw. Kl. II 18:1-72.

Stephen, M., and Cwilich, G. (1986) Rayleigh scattering and weak localization: Effects of polarization, Phys. Rev. B 34: 7564-7572.

Van Albada, A., and Lagendijk, A. (1985), Observations of weak localization of light in a random medium, Phys. Rev. Lett. 55:2692-2695.

Van Albada, A., Van der Mark, M,, and Lagendijk, A. (1987), Observation of weak localization of light in a finite slab: effects and light path classification, Phys. Rev. Lett. 58: 361-364.

Verstraete, M., Pinty, B., and Dickinson, R. (1990), A physical model of the bidirectional reflectance of vegetation canopies. 1. Theory, J. Geophys. Res. 95:11,755-11,765.

Woessner, P., and Hapke, B. (1987), Polarization of light scattered by clover, Remote Sens. Environ. 21:243-261.

Wolf, P., and Maret, G. (1985), Weak localization and coherent backscattering of photons in disordered media, Phys. Rev. Lett. 5:2696-2699. 